\title{
Site-specific Methylation of SST Gene May Serve as a Biomarker for Risk Prediction of Gastrointestinal Tract Cancers as Well as Promoting Malignant Behavior by Regulating Gene Expression
}

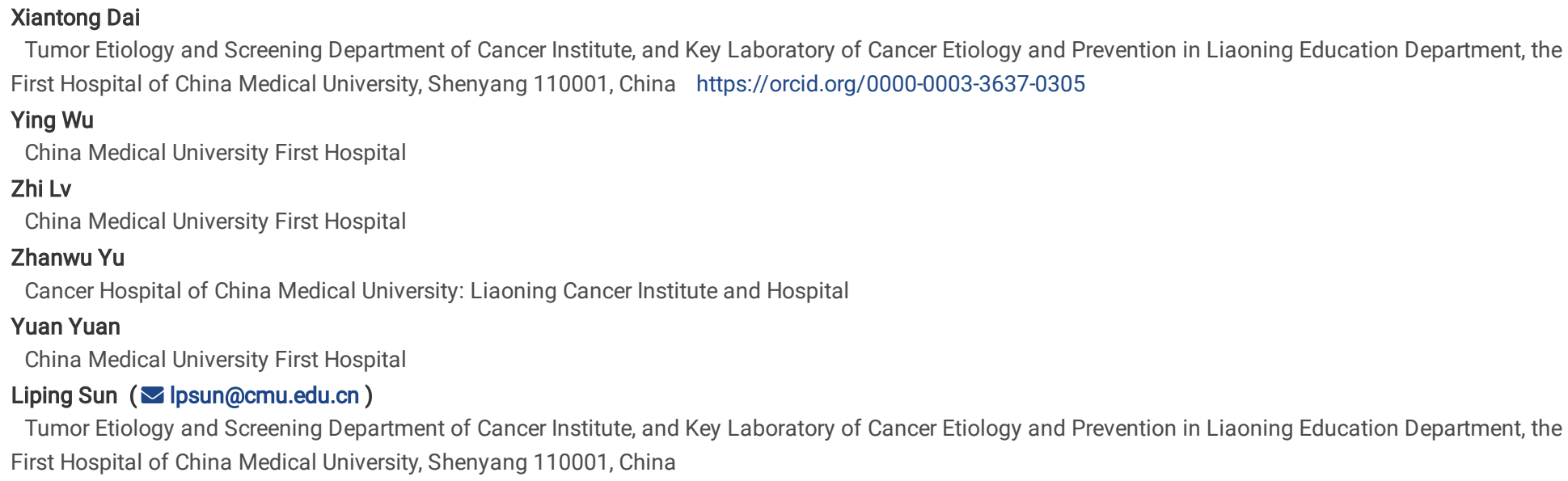




\section{Abstract}

Background: DNA methylation plays an important role in the regulation of gene expression as well as the occurrence and development of cancer. Abnormal hypermethylation in the promoter region of tumor suppressor genes is a recognized molecular events related to carcinogenesis. Somatostatin (SST) is considered as a tumor suppressor gene. The study aims to explore the relationship between the promoter methylation status of SST and the risk of gastrointestinal tract cancers and further to evaluate its diagnostic value.

Methods: Tissue samples were used for DNA isolation and RNA isolation. SST methylation was detected by biosulfite sequencing PCR, and SST mRNA expression was detected by quantitative real-time reverse transcription-polymerase chain reaction. The receiver operating curve (ROC) was drawn to evaluate the efficacy of SST CpG methylation on the diagnosis of digestive tract tumors by calculating the sensitivity, specificity and Youden index.

Results: The methylation level of SST promoter in cancer tissue were significantly increased compared with adjacent control tissue (EC: $0.649 \pm 0.078$ vs. $0.569 \pm 0.107, p<0.05, G C: 0.622 \pm 0.09$ vs. $0.588 \pm 0.079, p<0.05, C C: 0.663 \pm 0.083$ vs. $0.617 \pm 0.042 p<0.05)$. Significantly different methylation sites contained CpG $+18,+25,+44,+94,+100,+127$, and +129 . SST methylation status was associated with clinical phenotypes such as lymph node metastasis and vascular tumor thrombus. $\mathrm{CpG}$ sites in two tumors (+85 in EC, +94 in CC) were positively correlated with the depth of infiltration. The expression level of SST in gastric and colorectal cancer tissue was significantly lower than that in adjacent control tissue (both $p<0.001$ ). However, no statistically significant difference was found in EC group ( $p=0.32$ ). Except $\mathrm{CpG}+25$ and +85 sites in $\mathrm{GC}$ and the $+25,+85$ and +148 sites in $\mathrm{CRC}$, the methylation rate of each $\mathrm{CpG}$ was negatively correlated with gene expression (all $P<0.05$ ). Combined detection of eight CpG sites methylation $(+18,+42,+44,+85,+92,+94,+129$ and +138 ) showed the best area under the curve of 0.817 with sensitivity of $76.5 \%$ and specificity of $75 \%$.

Conclusions: Abnormal hypermethylation in SST promoter region increased the risk of gastrointestinal tract tumors, as well as promoting cancer progression by regulating gene expression. SST methylation status may serve as site-specific biomarker for risk prediction of gastrointestinal tract tumors.

\section{Introduction}

The gastrointestinal tract (GIT) including esophagus, stomach, and colorectum belong to tubular digestive organs. The tissue structures of GIT are highly similar, and also there are many similarities in the process of cancerization. Finding common molecular events involved in GIT cancers is helpful to interpret the cancerous pathway from the original source in GIT and also is helpful to identify tag-biomarker belong to GIT cancers. As we know that multiple genes and factors such as genetic variation and epigenetic changes participate in the occurrence and development of GIT cancers (1). DNA methylation is one of the most common epigenetic modification, mainly occurring at the fifth carbon atom of $\mathrm{CpG}$ island cytosine residue in the promoter region. Methylation in the promoter region of tumor suppressor genes leads to gene silencing by inhibiting gene transcription, thereby promoting the occurrence and development of tumors, affecting the risk and prognosis of tumors (2). DNA methylation is a reversible process (3), which commonly takes place in the early stage of tumorigenesis with the potential to be an early diagnostic cancer biomarker. Currently, the exploration of DNA methylation markers has become a hot spot in the epigenetics research of gastrointestinal tract tumors.

Growth hormone release inhibitory hormone (somatostatin, SST) is a member of the cyclic peptide family that has a broad inhibitory effect on the secretion of hormones such as growth hormone, insulin and glucagon. SST is located in human chromosome 3q27.3, and its protein somatostatin has two active forms, 14aa and 28aa, which are produced by the alternating cleavage of a single preproprotein encoded by the gene. SST has the effects of blocking the production of autocrine / paracrine growth-promoting hormone and growth factors, inhibiting growth factor-mediated mitotic signals and inducing apoptosis (4). It also inhibits the secretion of somatotropin, growth factors and has anti-angiogenic effects (5). These effects of directly or indirectly inhibiting tumorigenesis and development make SST have the potential to be a biomarker for detecting tumor risk. Our previous research has reported that SST is a core gene with high methylation and low expression in GC (6) and CRC (7). In addition, bioinformatics analysis results showed that SST in EC also had significant hypermethylation (data not shown). However, what is the correlation between the SST promoter methylation status and the risk of GIT cancers including esophageal cancer, gastric cancer, and colorectal cancer, and whether it plays a regulatory role by affecting gene expression levels, all of which remain to be further studied.

In this study, SST methylation status including average methylation, site-specific methylation, as well as mRNA expression level were detected in esophageal, gastric and colorectal cancers, simultaneously. On the basis, the efficacy of SST promoter methylation for diagnosis of GIT cancers was further evaluated. This research aimed at exploring comprehensively analyzed the effect of abnormal methylation in the promoter region of SST gene on the occurrence and development of GIT cancers by detecting methylation status and gene expression and clarify its potential as biomarker for GIT tumor risk. It is expected to provide valuable clues for revealing the possible mechanism of gastrointestinal tumors from the perspective of epigenetics, and to provide applicability signs for early diagnosis.

\section{Materials And Methods}

\subsection{Patients and Specimens}

The patients were recruited from the First Hospital of China Medical University and Cancer Hospital of China Medical University who underwent surgical resection without preoperative physical or chemical therapy from January 2013 to May 2018. Among them, 50 EC patients comprised of 46 males and 4 females with average age of 60 years (40-73); 99 GC patients comprised of 60 males and 31 females with average age of 60 years (25-83); 80 CRC patients comprised of 50 males and 30 females with average age of 61 years (30-79). All tissue samples utilized in this study were coded and collected from tissues that remained diagnosis. 
Tissue specimens including cancer and adjacent normal tissues were excised and directly put into RNAlater solution (RNAlater ${ }^{\text {TM }}$ Stabilization Solution, Thermo Fishe Scientifific, Waltham, MA, USA), and stored at $-80^{\circ} \mathrm{C}$ after $24 \mathrm{~h}$. Detailed clinical data were collected from the medical records of inpatients, including lymph node metastasis, vascular tumor thrombus, depth of infiltration and differentiation.

The current study was approved by the Human Ethics Review Committee of the First Hospital of China Medical University (Shenyang, China) and Cancer Hospital of China Medical University (Shenyang, China). Informed consent has been obtained from patients.

\subsection{DNA extraction}

SDS-phenol extraction method was used to organize tissue DNA. Took 0.1-0.2g of tissue, shredded and ground, added $400 \mu \mathrm{l}$ TE, $25 \mu \mathrm{l} 10 \%$ SDS and $10 \mu \mathrm{l} 20 \mathrm{mg}$ / $\mathrm{ml} \mathrm{PK}$ enzyme and mixed well. After $55^{\circ} \mathrm{C}$ water bath for $2 \mathrm{~h}$, phenol, chloroform and isoamyl alcohol mixture were used to remove protein. DNA was precipitated with $\mathrm{NaAc}$ and absolute ethanol and finally dissolved with TE solution.

\subsection{Biosulfite Sequence Polymerase chain reaction (BSP)}

SST promoter methylation was detected by BSP (Biosulfite Sequence Polymerase chain reaction). Sulfite modification of genomic DNA was performed using Zymo DNA Methylation-Gold kit (ZYMO). The Predict CpG islands tool in MethPrimer 2.0 (http://www.urogene.org/methprimer2) was employed to find the $\mathrm{CpG}$ island in SST promoter region and design nested PCR primers for methylated island. Outer primer sequences were forward 5'GTGTAATTGAGTGTGTATGTGTGGGAG-3' and reverse 5-ACAACAACCAAAAACTTCTACAAAAACTAAC-3'. Nested primer sequences were forward 5'AATGTGTATGTTTATAGTATTGAGTGA-3' and reverse 5'-AACACAACCCAAAACCAA-3'. Subsequently, PCR was carried out in a reaction condition of $94{ }^{\circ} \mathrm{C}$ for $10 \mathrm{~min},\left(94^{\circ} \mathrm{C} 20 \mathrm{~S}, 55^{\circ} \mathrm{C} 30 \mathrm{~S}, 72^{\circ} \mathrm{C} 30 \mathrm{~S}\right)$ for $40 \mathrm{cycles}, 72^{\circ} \mathrm{C}$ for $10 \mathrm{~min}$, and finally stored at $4{ }^{\circ} \mathrm{C}$. The nested PCR product was subjected to Sanger sequencing, and the sequencing primer was 5'-AGAGGGAGAYGGTTGAGA-3'. C and T signal values of CpG site were read respectively. The methylation rate of each site was calculated according to Meth\% $=C /(C+T) * 100 \%$.

\subsection{Total RNA extraction and reverse transcription}

Total RNA was extracted with TRIzol Reagent (TaKaRa, Dalian, China) from collected tissue. Rice-sized mucosa was digested with Trizol, and protein was removed with chloroform. RNA was precipitated with isopropanol and washed with 75\% ethanol. After ethanol was evaporated, RNA was dissolved with DEPC water and the Nano Drop was used to detect total RNA concentration. Oligo(dT) primers were used in the reverse-transcription step. The PrimeScript RT Master Mix (Takara) kit was used for reverse transcription.

\subsection{Quantitative Real-Time Reverse Transcription-Polymerase Chain Reaction (qRT-PCR)}

Realtime-PCR method was used to detect the relative expression of SST mRNA (TB Green Premix Ex Taq). And GAPDH was used as an internal reference to normalize SST expression. Primer sequences were listed as follows: SST, forward, 5'- CTGAACCCAACCAGACGGAG-3', reverse, 5'-GCCATAGCCGGGTTTGAGTT3'; GAPDH, forward, 5'-CCATCTTCCAGGAGCGAGATCCCT-3', reverse, 5'-CCTGCAAATGAGCCCCAGCC-3'. The reaction conditions were $95^{\circ} \mathrm{C} 30$ s, $\left(94^{\circ} \mathrm{C} 30\right.$ s, $56^{\circ} \mathrm{C} 20 \mathrm{~s}, 72^{\circ} \mathrm{C} 10 \mathrm{~s}$ ) for $40 \mathrm{cycles}$, and store at $4^{\circ} \mathrm{C} . \triangle \mathrm{Ct}$ and $2-\triangle \mathrm{Ct}$ values were calculated to evaluated the relative expression of SST.

\subsection{Statistical analysis}

Statistical analysis was performed using IBM SPSS Statistics 23 software and R 3.5.3. Paired T test was used to compare the difference in methylation and mRNA expression between cancer and control tissue. Spearman rank correlation was used to analyze the correlation between SST promoter methylation rate and its expression. Receiver operating curve (ROC) was adopted to evaluate diagnostic efficacy and calculate sensitivity (SEN), specificity (SPE) and Youden index (YD). $P<0.05$ was considered as statistical significance.

\section{Results}

\subsection{SST methylation rate in GIT cancers}

We detected the methylation rates of $15 \mathrm{CpG}$ sites, which were located in $+18,+25,+34,+42,+44,+85,+92,+94,+97,+100,+116,+127,+129,+138,+148$. (Figure 1)

The SST methylation rates in EC, GC, and CC were shown in Table 1, Figure 2.

In 42 cases of EC, the average methylation rate in cancer tissue of EC was significantly higher than that in control tissue ( $p<0.05)$, including the $+18,+34,+42,+44,+92,+94,+100,+116,+127,+129,+138$ and +148 CpG sites $(p<0.05)$.

In 99 cases of GC, the average methylation rate in cancer tissue was significantly higher than that in control tissue $(p<0.05)$, including the $+18,+42,+44$, $+94,+100,+127$ and +129 CpG sites (all $p<0.05)$.

In 70 cases of CC, the average methylation rate in cancer tissue was significantly 
higher than that in control tissue $(p<0.05)$, including the $+18,+34,+42,+44,+92,+94,+97,+100,+116,+127,+129,+138$ and +148 CpG sites $($ all $p<0.05)$.

Joint analysis for the methylation difference of $\mathrm{CpG}$ sites in SST promoters for the three cancers showed that the methylation rates of $\mathrm{CpG}$ sites 7 sites including $+18,+42,+44,+94,+100,+127$, and +129 were meaningful significantly different from the adjacent control tissue. However, but the differences of CpG sites +25 and +85 were not statistically significant. The CpG sites of $+34,+92$ and +116 were significantly different in EC and CC, however, no significant difference was observed in GC. The CpG site +97 was significantly different in CC but not in EC and GC. The CpG sites +138 and +148 were significantly different in EC but not in the others.

Table 1 Methylation rates of CpG sites in GIT cancers

\begin{tabular}{|c|c|c|c|c|c|c|c|c|c|}
\hline \multirow[t]{2}{*}{ CpG site } & \multicolumn{6}{|l|}{ Mean \pm Std } & \multicolumn{3}{|l|}{$\mathrm{P}$ Value } \\
\hline & EC & EN & GC & $\mathrm{GN}$ & CRC & CRN & EC/EN & $\mathrm{GC} / \mathrm{GN}$ & CRC/CRN \\
\hline 9 & $0.457 \pm 0.157$ & $0.317 \pm 0.105$ & $0.416 \pm 0.116$ & $0.359 \pm 0.111$ & $0.425 \pm 0.126$ & $0.374 \pm 0.104$ & $<0.001$ & $<0.001$ & 0.005 \\
\hline 10 & $0.495 \pm 0.136$ & $0.45 \pm 0.132$ & $0.494 \pm 0.097$ & $0.482 \pm 0.12$ & $0.495 \pm 0.097$ & $0.475 \pm 0.102$ & 0.129 & 0.404 & 0.182 \\
\hline 11 & $0.521 \pm 0.154$ & $0.416 \pm 0.131$ & $0.51 \pm 0.102$ & $0.486 \pm 0.089$ & $0.537 \pm 0.105$ & $0.488 \pm 0.071$ & 0.003 & 0.098 & 0.001 \\
\hline 12 & $0.53 \pm 0.15$ & $0.438 \pm 0.143$ & $0.556 \pm 0.123$ & $0.492 \pm 0.091$ & $0.607 \pm 0.119$ & $0.526 \pm 0.053$ & 0.004 & $<0.001$ & $<0.001$ \\
\hline 13 & $0.541 \pm 0.158$ & $0.438 \pm 0.146$ & $0.545 \pm 0.144$ & $0.489 \pm 0.101$ & $0.614 \pm 0.122$ & $0.529 \pm 0.055$ & 0.002 & 0.002 & $<0.001$ \\
\hline 14 & $0.483 \pm 0.121$ & $0.469 \pm 0.117$ & $0.479 \pm 0.122$ & $0.474 \pm 0.091$ & $0.518 \pm 0.101$ & $0.52 \pm 0.073$ & 0.565 & 0.734 & 0.9 \\
\hline 15 & $0.72 \pm 0.098$ & $0.668 \pm 0.117$ & $0.678 \pm 0.11$ & $0.669 \pm 0.085$ & $0.74 \pm 0.099$ & $0.708 \pm 0.067$ & 0.032 & 0.551 & 0.019 \\
\hline 16 & $0.664 \pm 0.125$ & $0.57 \pm 0.136$ & $0.62 \pm 0.132$ & $0.576 \pm 0.099$ & $0.698 \pm 0.116$ & $0.62 \pm 0.081$ & 0.002 & 0.009 & $<0.001$ \\
\hline 17 & $0.575 \pm 0.129$ & $0.538 \pm 0.127$ & $0.58 \pm 0.138$ & $0.547 \pm 0.1$ & $0.646 \pm 0.122$ & $0.576 \pm 0.075$ & 0.188 & 0.061 & $<0.001$ \\
\hline 18 & $0.624 \pm 0.111$ & $0.568 \pm 0.121$ & $0.602 \pm 0.132$ & $0.568 \pm 0.106$ & $0.691 \pm 0.111$ & $0.623 \pm 0.067$ & 0.017 & 0.041 & $<0.001$ \\
\hline 19 & $0.781 \pm 0.095$ & $0.701 \pm 0.118$ & $0.715 \pm 0.088$ & $0.69 \pm 0.088$ & $0.765 \pm 0.098$ & $0.722 \pm 0.067$ & 0.001 & 0.061 & 0.002 \\
\hline 20 & $0.808 \pm 0.09$ & $0.703 \pm 0.126$ & $0.744 \pm 0.103$ & $0.705 \pm 0.097$ & $0.774 \pm 0.094$ & $0.73 \pm 0.069$ & $<0.001$ & 0.009 & 0.001 \\
\hline 21 & $0.827 \pm 0.091$ & $0.708 \pm 0.131$ & $0.765 \pm 0.104$ & $0.711 \pm 0.105$ & $0.799 \pm 0.095$ & $0.753 \pm 0.063$ & $<0.001$ & 0.001 & 0.001 \\
\hline 22 & $0.865 \pm 0.067$ & $0.796 \pm 0.115$ & $0.825 \pm 0.08$ & $0.804 \pm 0.086$ & $0.838 \pm 0.076$ & $0.822 \pm 0.067$ & 0.001 & 0.102 & 0.111 \\
\hline 23 & $0.839 \pm 0.083$ & $0.761 \pm 0.116$ & $0.788 \pm 0.087$ & $0.77 \pm 0.091$ & $0.804 \pm 0.096$ & $0.793 \pm 0.066$ & 0.001 & 0.186 & 0.325 \\
\hline AVG & $0.649 \pm 0.078$ & $0.569 \pm 0.107$ & $0.622 \pm 0.09$ & $0.588 \pm 0.079$ & $0.663 \pm 0.083$ & $0.617 \pm 0.042$ & $<0.001$ & 0.008 & $<0.001$ \\
\hline
\end{tabular}

\subsection{Correlation of methylation of SST and tumor biological behavior}

We analyzed the methylation rate of SST promoter region in four tumor biological behaviors (Lymph node metastasis, Vascular tumor thrombus, Depth of infiltration and Differentiation). (Table 2)

In EC, two sites $(+127,+129)$ were different in vascular tumor thrombus $(0.861 \pm 0.071$ vs. $0.794 \pm 0.090 p=0.021,0.878 \pm 0.068$ vs. $0.813 \pm 0.093 p=0.038)$. The methylation rates of the above two $\mathrm{CpG}$ sites in positive vascular cancer thrombus group was significantly higher than that in negative group. In the depth of infiltration, the muscular layer methylation rate at site +85 was the lowest, $0.422 \pm 0.105$, serous layer was $0.507 \pm 0.117$, and mucosa and submucosa were $0.534 \pm 0.172(p=0.041)$. At site +18 , the methylation rate of poor differentiation was $0.616 \pm 0.137$, and medium and high differentiation were $0.432 \pm 0.153$ and $0.442 \pm 0.145$ respectively $(p=0.014)$.

In $\mathrm{GC}$, the methylation rate at site +25 was related to lymph node metastasis, and the positive group was significantly lower than the negative group $(0.477 \pm 0.092$ vs. $0.536 \pm 0.103 p=0.013)$.

In $\mathrm{CC}$, the methylation rate at position +94 was related to the depth of infiltration, and the serous layer was significantly higher than the muscular layer $(0.718 \pm 0.098$ vs. $0.649 \pm 0.129 p=0.025)$. In this experiment, the infiltration depth of colorectal cancer did not have cases below the muscular layer.

\subsection{SST mRNA expression in GIT cancers}

The SST mRNA expressions in EC, GC, and CC were shown in Figure 3. 
In 50 cases of EC, no statistically significant difference was found in SST mRNA expression between cancer and adjacent control tissue ( $0.0167 \pm 0.0455$ vs. $0.033 \pm 0.1061, p=0.32)$.

In 52 cases of GC, the expression of SST mRNA in cancer tissue was significantly lower than that in adjacent control tissue ( $0.0086 \pm 0.0176$ vs. $0.0318 \pm$ $0.0404, p<0.001$ );

In 65 cases of CC, the expression of SST mRNA in cancer tissue was significantly lower than that in adjacent control tissue (0.0098 \pm 0.0263 vs. $0.0819 \pm$ $0.1372, p<0.001)$.

\subsection{Correlation between SST promoter methylation and mRNA expression in GIT cancers}

SST promoter methylation and SST mRNA expression had no significant correlation in EC group. In GC group, except CpG sites +25 and +85 , the SST methylation and expression of other sites were negatively correlated $(P<0.05)$. For CC group, except CpG sites of $+25,+85$ and +148 , methylation rate was negatively correlated with expression $(P<0.05)$. (Table 3$)$

Table 2 Correlation analysis of methylation of SST promoter region and tumor biological behavior of GIT cancers 


\begin{tabular}{|c|c|c|c|c|c|c|c|c|c|c|c|c|c|c|c|}
\hline Cancer & Parameter & Group & $\mathbf{n}$ & +18 & +25 & +34 & +42 & +44 & +85 & +92 & +94 & +97 & +100 & +116 & +127 \\
\hline \multirow[t]{10}{*}{ EC } & $\begin{array}{l}\text { Lymphatic } \\
\text { metastasis }\end{array}$ & Positive & 25 & 0.497 & 0.654 & 0.71 & 0.311 & 0.405 & 0.858 & 0.617 & 0.749 & 0.798 & 0.265 & 0.405 & 0.828 \\
\hline & & Negetive & 17 & & & & & & & & & & & & \\
\hline & $\begin{array}{l}\text { Vessel } \\
\text { carcinoma } \\
\text { embolus }\end{array}$ & Positive & 9 & 0.718 & 0.976 & 0.786 & 0.587 & 0.414 & 0.249 & 0.526 & 0.651 & 0.303 & 0.171 & 0.303 & 0.021 \\
\hline & & Negetive & 33 & & & & & & & & & & & & \\
\hline & $\begin{array}{l}\text { Depth of } \\
\text { infiltration }\end{array}$ & serosa & 26 & 0.141 & 0.451 & 0.92 & 0.201 & 0.268 & 0.041 & 0.478 & 0.668 & 0.099 & 0.035 & 0.094 & 0.4 \\
\hline & & muscular & 13 & & & & & & & & & & & & \\
\hline & & $\begin{array}{l}\text { mucosal } \\
\text { and } \\
\text { Submucosal }\end{array}$ & 3 & & & & & & & & & & & & \\
\hline & Differentiation & poorly & 5 & 0.014 & 0.17 & 0.15 & 0.381 & 0.381 & 0.827 & 0.937 & 0.897 & 0.803 & 0.977 & 0.195 & 0.289 \\
\hline & & moderately & 21 & & & & & & & & & & & & \\
\hline & & high & 16 & & & & & & & & & & & & \\
\hline \multirow[t]{10}{*}{ GC } & $\begin{array}{l}\text { Lymphatic } \\
\text { metastasis }\end{array}$ & Positive & 64 & 0.471 & 0.013 & 0.073 & 0.257 & 0.578 & 0.339 & 0.821 & 0.804 & 0.91 & 0.958 & 0.566 & 0.869 \\
\hline & & Negetive & 27 & & & & & & & & & & & & \\
\hline & $\begin{array}{l}\text { Vessel } \\
\text { carcinoma } \\
\text { embolus }\end{array}$ & Positive & 57 & 0.961 & 0.993 & 0.085 & 0.608 & 0.426 & 0.348 & 0.724 & 0.931 & 0.879 & 0.583 & 0.688 & 0.737 \\
\hline & & Negetive & 34 & & & & & & & & & & & & \\
\hline & $\begin{array}{l}\text { Depth of } \\
\text { infiltration }\end{array}$ & serosa & 79 & 0.472 & 0.662 & 0.743 & 0.508 & 0.347 & 0.097 & 0.485 & 0.668 & 0.284 & 0.517 & 0.309 & 0.479 \\
\hline & & muscular & 9 & & & & & & & & & & & & \\
\hline & & $\begin{array}{l}\text { mucosal } \\
\text { and } \\
\text { Submucosal }\end{array}$ & 3 & & & & & & & & & & & & \\
\hline & Differentiation & poorly & 75 & 0.608 & 0.658 & 0.811 & 0.725 & 0.667 & 0.119 & 0.775 & 0.991 & 0.551 & 0.884 & 0.446 & 0.499 \\
\hline & & moderately & 12 & & & & & & & & & & & & \\
\hline & & high & 4 & & & & & & & & & & & & \\
\hline \multirow[t]{10}{*}{ CRC } & $\begin{array}{l}\text { Lymphatic } \\
\text { metastasis }\end{array}$ & Positive & 38 & 0.658 & 0.56 & 0.939 & 0.81 & 0.923 & 0.758 & 0.721 & 0.973 & 0.758 & 0.651 & 0.21 & 0.272 \\
\hline & & Negetive & 42 & & & & & & & & & & & & \\
\hline & $\begin{array}{l}\text { Vessel } \\
\text { carcinoma } \\
\text { embolus }\end{array}$ & Positive & 11 & 0.917 & 0.273 & 0.743 & 0.939 & 0.994 & 0.227 & 0.534 & 0.905 & 0.85 & 0.917 & 0.553 & 0.66 \\
\hline & & Negetive & 69 & & & & & & & & & & & & \\
\hline & $\begin{array}{l}\text { Depth of } \\
\text { infiltration }\end{array}$ & serosa & 59 & 0.581 & 0.891 & 0.267 & 0.184 & 0.102 & 0.9 & 0.13 & 0.025 & 0.184 & 0.083 & 0.119 & 0.058 \\
\hline & & muscular & 21 & & & & & & & & & & & & \\
\hline & & $\begin{array}{l}\text { mucosal } \\
\text { and } \\
\text { Submucosal }\end{array}$ & 0 & & & & & & & & & & & & \\
\hline & Differentiation & poorly & 25 & 0.76 & 0.36 & 0.53 & 0.289 & 0.151 & 0.519 & 0.24 & 0.081 & 0.351 & 0.271 & 0.426 & 0.139 \\
\hline & & moderately & 40 & & & & & & & & & & & & \\
\hline & & high & 15 & & & & & & & & & & & & \\
\hline
\end{tabular}




\begin{tabular}{|lllllll|}
\hline CpG site & $\mathbf{r}$ & \multicolumn{5}{l|}{$\mathbf{P}$} \\
\cline { 2 - 7 }+18 & EC & GC & CC & EC & GC & CC \\
\hline+25 & -0.063 & -0.363 & -0.23 & 0.57 & $<0.001$ & 0.008 \\
\hline+34 & -0.13 & -0.154 & -0.17 & 0.239 & 0.118 & 0.053 \\
\hline+42 & -0.071 & -0.271 & -0.23 & 0.518 & 0.005 & 0.008 \\
\hline+44 & 0.005 & -0.393 & -0.379 & 0.966 & $<0.001$ & $<0.001$ \\
\hline+85 & 0.026 & -0.371 & -0.396 & 0.816 & $<0.001$ & $<0.001$ \\
\hline+92 & -0.025 & -0.107 & 0.083 & 0.818 & 0.28 & 0.346 \\
\hline+94 & -0.003 & -0.355 & -0.406 & 0.975 & $<0.001$ & $<0.001$ \\
\hline+97 & 0.083 & -0.252 & -0.36 & 0.453 & 0.01 & $<0.001$ \\
\hline+100 & 0.048 & -0.211 & -0.361 & 0.663 & 0.031 & $<0.001$ \\
\hline+116 & -0.089 & -0.252 & -0.273 & 0.419 & 0.01 & 0.002 \\
\hline+127 & -0.034 & -0.255 & -0.272 & 0.758 & 0.009 & 0.002 \\
\hline+129 & -0.047 & -0.334 & -0.306 & 0.668 & 0.001 & $<0.001$ \\
\hline+138 & 0.074 & -0.292 & -0.179 & 0.501 & 0.003 & 0.041 \\
\hline+148 & 0.045 & -0.212 & -0.129 & 0.687 & 0.031 & 0.144 \\
\hline AVG & -0.026 & -0.35 & -0.374 & 0.813 & $<0.001$ & $<0.001$ \\
\hline
\end{tabular}

\subsection{The efficacy of SST promoter methylation for diagnosis of GIT cancers}

Multivariate logistic regression was used to build appropriate diagnostic models. The diagnostic efficiency was assessed with sensitivity, specificity, Yorden Index and AUC of ROC. The combination of $8 \mathrm{CpG}$ sites $(+18,+42,+44,+85,+92,+94,+129,+138)$ had the largest area under the curve (AUC) of 0.817 with a sensitivity of $76.5 \%$ and a specificity of $75.0 \%$ GIT cancers. For GIT cancers, upper gastrointestinal tract, gastrointestinal, EC, GC, and CC models were established with AUC above 0.8. Co-differential sites that were significantly negatively correlated with expression in the first model were selected for modeling. In EC, the combination of 2 CpG sites $(+18,+129)$ had the best diagnostic efficiency of AUC at 0.818 with a sensitivity of $80.0 \%$ and a specificity of $72.3 \%$. (Table 4, Figure 4)

Table 4 Diagnostic value of combinations of $\mathrm{CpG}$ sites 


\begin{tabular}{|lllllll|}
\hline CpG sites & Tumor & AUC & Sen & Spe & YD & Cut off \\
\hline$+18,+42,+44,+85,+92$, & Gastrointestinal tract tumor & 0.817 & 0.765 & 0.75 & 0.515 & $50 \%$ \\
$+94,+129,+138$ & & & & & & \\
\hline$+18,+92,+94,+129,+138$ & Upper digestive tract tumor & 0.816 & 0.681 & 0.849 & 0.53 & $57.20 \%$ \\
$+42,+44,+85,+92,+94$, & Gastrointestinal tumor & 0.819 & 0.685 & 0.865 & 0.55 & $58.10 \%$ \\
$+116,+129,+138$ & & & & & & \\
$++18,+25,+97,+129$ & Esophageal cancer & 0.868 & 0.778 & 0.83 & 0.608 & $50.20 \%$ \\
\hline$+18,+92,+129,+148$ & Gastric cancer & 0.812 & 0.657 & 0.838 & 0.495 & $57.40 \%$ \\
\hline$+44,+92,+94$ & Colorectal cancer & 0.813 & 0.756 & 0.779 & 0.535 & $50.10 \%$ \\
\hline$+18,+129$ & Gastrointestinal tract tumor & 0.713 & 0.65 & 0.681 & 0.331 & $49.70 \%$ \\
\hline$+18,+129$ & Upper digestive tract tumor & 0.723 & 0.667 & 0.671 & 0.338 & $48.10 \%$ \\
\hline+42 & Gastrointestinal tumor & 0.712 & 0.492 & 0.892 & 0.384 & $56.60 \%$ \\
\hline$+18,+129$ & Esophageal cancer & 0.818 & 0.8 & 0.723 & 0.523 & $39.90 \%$ \\
\hline$+42,+129$ & Gastric cancer & 0.682 & 0.576 & 0.727 & 0.303 & $52.80 \%$ \\
\hline$+44,+94$ & Colorectal cancer & 0.796 & 0.732 & 0.814 & 0.546 & $49.40 \%$ \\
\hline
\end{tabular}

\section{Discussion}

The decrease of tumor suppressor gene expression is an important event of carcinogenesis process. Hypermethylation of DNA is related to promoter activity and can be secondary to trigger gene (tumor suppressor gene) silencing. Differences in gene expression caused by the methylation status of transcription factor binding sites in promoter region are important molecular mechanism for regulation of tumorigenesis and development (8). In this study, EC, GC, and CC tissue samples were used to detect the methylation status of $15 \mathrm{CpG}$ sites in the SST promoter region in GIT cancers. We found that the hypermethylation status of SST promoter region was positively correlated with the risk of GIT cancers EC,GC and CC. The combination of 8 CpG sites $(+18,+42,+44,+85,+92,+94,+129,+138)$ had the largest AUC of 0.817 with a SEN of $76.5 \%$ and a SPE of $75.0 \%$ for the three cancers, suggesting that SST methylation status may serve as a site-specific biomarker for risk prediction of GIT cancers. In addition, several common significant hypermethylation sites including CpG $+85,+97$, and +100 were significantly positively correlated with the depth of tumor invasion. Hypermethylation of SST promoter in GIT cancers was significantly negatively correlated with mRNA expression level, suggesting that SST abnormal hypermethylation can affect the occurrence and development of GIT cancers by inhibiting gene transcription. In addition, the combination of 8 CpG sites $(+18,+42,+44,+85,+92,+94,+129,+138)$ had the largest AUC of 0.817 with a SEN of $76.5 \%$ and a SPE of $75.0 \%$ for the three cancers. This study provided applicability markers for the combined diagnosis of GIT cancers and also valuable clues for analyzing the possible mechanism of GIT cancers from an epigenetic perspective.

Previous studies have reported that abnormal methylation of APC and $\mathrm{CDH} 1$ genes is associated with gastric cancer and colorectal cancer risk (9). CDH4 gene exhibits hypermethylation in 78\% colorectal cancer and 95\% gastric cancer tissue (10). RUNX3 hypermethylation is significantly linked to the risk of esophageal cancer (11), gastric cancer (12)and colorectal cancer(13). In the present study, we quantitatively detected the methylation rates of SST promoter region in GIT cancers and the results showed that the methylation rate of SST promoter region in EC.GC and CC were significantly higher than that of adjacent control tissue, with some common differential CpG sites. On the basis, we conducted a conjoint analysis for the efficacy of SST methylation-specific CpG sites in the diagnosis of GIT cancers. The results showed that multi-site combined diagnosis of esophageal and colorectal cancer had better SEN and SPE (83.3\%, $69.0 \%, 63.1 \%$, and $89.2 \%)$, CpG +42 has a higher SPE (91.2\%) for gastric cancer, and SEN $50 \%$ and SET $87.9 \%$ for the combined diagnosis of triple cancer, suggesting that SST abnormal methylation has a potential role of diagnosing GIT cancers. Recent studies have suggested that abnormally high methylation of DNA promoters can be used as diagnostic biomarkers for lung cancer (14), colorectal cancer (15), pancreatic cancer(16) and gastric cancer (17) , playing an important role in the screening and diagnosis of digestive tumors (18) (19) (20). This study identified a new methylation marker SST that was significantly related to the risk of digestive tract GIT cancers. Future research can be used as a basis to analyze the role of methylation regulation and explore the value of clinical application.

Hypermethylation of promoter is one of the earliest somatic genome changes that occur during the development of various cancers (21).The abnormal methylation status at specific sites is more closely related to malignant biological behavior of tumors. This study found that three $\mathrm{CpG}$ sites of esophageal cancer increased with T stage. The methylation status of different $\mathrm{CpG}$ sites was related to the depth of vascular tumor thrombus and invasion. The methylation status of multiple CpG sites in gastric cancer was related to clinicopathological parameters of gender, infiltration depth, lymph node metastasis and Lauren classification. Colorectal cancer $\mathrm{CpG}$ sites methylation status was significantly related to age, gender, T stage, growth pattern, infiltration depth and other parameters. The $+85,+97$ and $+100 \mathrm{CpG}$ sites are the common sites related to the depth of gastrointestinal tumor invasion. The $+85 \mathrm{CpG}$ site methylation was related to multiple clinicopathological parameters while had no significant difference between cancer and normal control tissue, suggesting the limited role of +85 site in tumorigenesis but the significant role in tumor development. Previous studies have shown that although suppressor genes often show a high methylation status in the promoter region in tumors, not every $\mathrm{CpG}$ sites have abnormally high methylation status. Some sites even show low methylation status. Abnormal hypermethylation mainly affects cell biological function and DNA expression by inhibiting the binding of transcription factors to the promoter region, thereby promoting tumorigenesis. Zou B et al. found in the study of gastric cancer and colorectal cancer that the silencing of XAF1 gene 3 


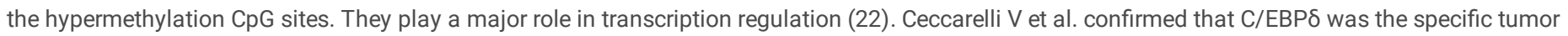
suppressor gene in leukemia. C/ EBPS gene re-expression is regulated by a single CpG site demethylation. An in-depth functional study of the above SSTspecific CpG sites is expected to analyze the molecular mechanism of SST abnormal methylation involved in the development of gastrointestinal tumors.

At present, among the studies about SST expression in gastrointestinal tract tumors, Jackson K et al. found that 93\% gastric cancer samples had downregulated SST transcription(19). Leiszter K et al. pointed out that the expression level of SST in colorectal cancer tissue decreased significantly compared with normal control tissue. The expression of SST in esophagus has not been reported. SST is mainly secreted by D cells in brain and gastric antrum, and is expressed throughout the body, with higher levels expressed in stomach, intestine and brain tissue. In this study, the expression levels of SST in the three cancers showed different levels of down-regulation, among which gastric cancer and colorectal cancer were significantly down-regulated, while the difference in esophageal cancer tissue did not have statistical significance $(P=0.32)$. We speculated that it might be due to the low expression level of SST in esophageal tissue. Therefore, future research on SST and esophageal cancer risk needs to be further studied. As a neurotransmitter, SST is an inhibitor of hormone and growth factor secretion, which can inhibit the release of gastric acid, pepsin, gastrin, etc., reduce gastrointestinal secretion and inhibit the growth of digestive tract cells. It also inhibits growth hormone, thyroid stimulating hormone and the secretion of insulin and glucagon. SST maintains the stability of the above hormone levels in the body. Recent studies have found that SST can also be used as an immunomodulator to inhibit cell proliferation under specific conditions (4). SST blocks the production of autocrine / paracrine growth-promoting hormones and growth factors. On the one hand, it can inhibit cell proliferation by inhibiting growth factor-mediated mitotic signals and inducing apoptosis (23). On the other hand, it can also inhibit the production and growth of tumor cells by the effect of inhibiting the secretion of growth hormone and growth factor and anti-angiogenesis indirectly (24). Therefore, SST production is suppressed and expression is reduced, causing abnormal increase in secretion of growth hormone and growth factor. Then the secretion of digest tract hormone increases and promotes the growth, mitosis and proliferation of cells in the digestive tract. Thus SST has an inhibitory effect on the occurrence and development of gastrointestinal tract tumors. The results of this study confirmed that the down-regulation of SST expression was an important factor in the development of gastrointestinal tumors. It remains to be clarified whether it is related to the risk of esophageal cancer.

The correlation analysis between the methylation status of SST promoter region and mRNA expression showed that multiple CpG sites and average methylation rate in gastric cancer and colorectal cancer were significantly negatively correlated with SST expression. Usually, the CpG site of gene promoter region is methylated, and the addition of a new methyl group to the cytosine 5 carbon atom causes the chromatin conformation of this region to change, which in turn blocks the interaction of transcription factors with this region, inhibiting gene transcription, reducing protein expression and affecting the normal biological function of cells (25). It is an important epigenetic regulatory factor for tumorigenesis and development. We took advantage of bioinformatics to predict and analyze that the common differences methylated $\mathrm{CpG}$ sites +42 and +44 of three tumors were the binding sites of transcription factors RHOXF1, ETS1, GSC, GSC2, DPRX, OTX1 and OTX2. The CpG sites +127 and +129 were the binding sites of transcription factors EBF1 and NR2C2. We speculated that during the malignant transformation of digestive tract epithelial cells, the abnormally high methylation status of the specific CpG site in the promoter region of SST gene may inhibit the transcription of SST gene and reduce the expression of mRNA by inhibiting the binding of a transcription factor to the transcription binding site, thereby promoting the occurrence and development of tumors. The above hypothesis needs to be confirmed by in-depth molecular biology experiments.

In summary, our study suggested that the abnormal hypermethylation in SST promoter region increased the risk of GIT cancers. gastrointestinal tract tumors. It may promote the cancergenesis and cancer progression by inhibiting gene transcription. The site-specific methylation status in SST may serve as a potential biomarker for risk prediction of GIT cancers. gastrointestinal tract tumors. The abnormal methylation status at specific sites is more closely related to malignant biological behavior of GIT cancers.

\section{Abbreviations}

SST somatostatin

ROC The receiver operating curve

CpG cytosines followed by guanine residues

EC esophageal cancer

GC gastric cancer

CC colorectal cancer

GIT gastrointestinal tract

TE Tris-EDTA buffer solution

BSP Biosulfite Sequence Polymerase chain reaction

SEN sensitivity

SPE specificity

YD Youden index 


\section{Declarations}

Ethics approval and consent to participate: The study was approved by the Ethics Review Committee of China Medical University (Shenyang, China).

Consent for publication: Not applicable

Availability of data and materials: The datasets used and/or analyzed during the current study are available from the corresponding author on reasonable request.

Competing interests: The authors declare no conflicts of interest.

Funding: This work was funded by grants from the National Key R\&D Program of China (Grant 2018YFC1311600) and the Liaoning Provence Key R\&D Program (Grant 2020JH2/10300063).

Authors' contributions: DXT and SLP designed the study; DXT, SLP, LZ, and YZ performed the experiments and prepared the figures; SLP, YY, and LZ contributed to drafting the manuscript. All authors read and approved the final manuscript.

Acknowledgements: Not applicable

\section{References}

1. Bijlsma MF, Sadanandam A, Tan P, Vermeulen L. Molecular subtypes in cancers of the gastrointestinal tract. Nature reviews Gastroenterology \& hepatology. 2017;14(6):333-42.

2. Greenberg MVC, Bourc'his D. The diverse roles of DNA methylation in mammalian development and disease. Nature reviews Molecular cell biology. 2019;20(10):590-607.

3. Wu X, Zhang Y. TET-mediated active DNA demethylation: mechanism, function and beyond. Nature reviews Genetics. 2017;18(9):517-34.

4. O'Toole TJ, Sharma S. Physiology, Somatostatin. StatPearls. Treasure Island (FL): StatPearls Publishing Copyright @ 2020, StatPearls Publishing LLC.; 2020.

5. Weckbecker G, Lewis I, Albert R, Schmid HA, Hoyer D, Bruns C. Opportunities in somatostatin research: biological, chemical and therapeutic aspects. Nature reviews Drug discovery. 2003;2(12):999-1017.

6. Li H, Liu JW, Liu S, Yuan Y, Sun LP. Bioinformatics-Based Identification of Methylated-Differentially Expressed Genes and Related Pathways in Gastric Cancer. Digestive diseases and sciences. 2017;62(11):3029-39.

7. Liu J, Li H, Sun L, Wang Z, Xing C, Yuan Y. Aberrantly methylated-differentially expressed genes and pathways in colorectal cancer. Cancer cell international. 2017;17:75.

8. Tahara T, Arisawa T. Potential usefulness of DNA methylation as a risk marker for digestive cancer associated with inflammation. Expert review of molecular diagnostics. 2012;12(5):489-97.

9. Coppedè F. Epigenetic biomarkers of colorectal cancer: Focus on DNA methylation. Cancer letters. 2014;342(2):238-47.

10. Miotto E, Sabbioni S, Veronese A, Calin GA, Gullini S, Liboni A, et al. Frequent aberrant methylation of the CDH4 gene promoter in human colorectal and gastric cancer. Cancer research. 2004;64(22):8156-9.

11. Long C, Yin B, Lu Q, Zhou X, Hu J, Yang Y, et al. Promoter hypermethylation of the RUNX3 gene in esophageal squamous cell carcinoma. Cancer investigation. 2007;25(8):685-90.

12. Kaise M, Yamasaki T, Yonezawa J, Miwa J, Ohta Y, Tajiri H. CpG island hypermethylation of tumor-suppressor genes in H. pylori-infected non-neoplastic gastric mucosa is linked with gastric cancer risk. Helicobacter. 2008;13(1):35-41.

13. Imamura Y, Hibi K, Koike M, Fujiwara M, Kodera Y, Ito K, et al. RUNX3 promoter region is specifically methylated in poorly-differentiated colorectal cancer. Anticancer research. 2005;25(4):2627-30.

14. Belinsky SA. Gene-promoter hypermethylation as a biomarker in lung cancer. Nature reviews Cancer. 2004;4(9):707-17.

15. Ng JM, Yu J. Promoter hypermethylation of tumour suppressor genes as potential biomarkers in colorectal cancer. International journal of molecular sciences. 2015;16(2):2472-96.

16. Tang B, Li Y, Qi G, Yuan S, Wang Z, Yu S, et al. Clinicopathological Significance of CDKN2A Promoter Hypermethylation Frequency with Pancreatic Cancer. Scientific reports. 2015;5:13563.

17. Corso G, Pedrazzani C, Pinheiro H, Fernandes E, Marrelli D, Rinnovati A, et al. E-cadherin genetic screening and clinico-pathologic characteristics of early onset gastric cancer. European journal of cancer (Oxford, England : 1990). 2011;47(4):631-9.

18. Ushiku H, Yamashita K, Ema A, Minatani N, Kikuchi M, Kojo K, et al. DNA diagnosis of peritoneal fluid cytology test by CDO1 promoter DNA hypermethylation in gastric cancer. Gastric cancer : official journal of the International Gastric Cancer Association and the Japanese Gastric Cancer Association. 2017;20(5):784-92.

19. Jin Z, Olaru A, Yang J, Sato F, Cheng Y, Kan T, et al. Hypermethylation of tachykinin-1 is a potential biomarker in human esophageal cancer. Clinical cancer research : an official journal of the American Association for Cancer Research. 2007;13(21):6293-300.

20. Gerecke C, Scholtka B, Löwenstein Y, Fait I, Gottschalk U, Rogoll D, et al. Hypermethylation of ITGA4, TFPI2 and VIMENTIN promoters is increased in inflamed colon tissue: putative risk markers for colitis-associated cancer. Journal of cancer research and clinical oncology. 2015;141(12):2097-107. 
21. Bastian PJ, Yegnasubramanian S, Palapattu GS, Rogers CG, Lin X, De Marzo AM, et al. Molecular biomarker in prostate cancer: the role of CpG island hypermethylation. European urology. 2004;46(6):698-708.

22. Zou B, Chim CS, Zeng H, Leung SY, Yang Y, Tu SP, et al. Correlation between the single-site CpG methylation and expression silencing of the XAF1 gene in human gastric and colon cancers. Gastroenterology. 2006;131(6):1835-43.

23. Rorsman P, Huising MO. The somatostatin-secreting pancreatic 8 -cell in health and disease. Nature reviews Endocrinology. 2018;14(7):404-14.

24. Rai U, Thrimawithana TR, Valery C, Young SA. Therapeutic uses of somatostatin and its analogues: Current view and potential applications. Pharmacology \& therapeutics. 2015;152:98-110.

25. Eigler T, Ben-Shlomo A. Somatostatin system: molecular mechanisms regulating anterior pituitary hormones. Journal of molecular endocrinology. 2014;53(1):R1-19.

\section{Figures}

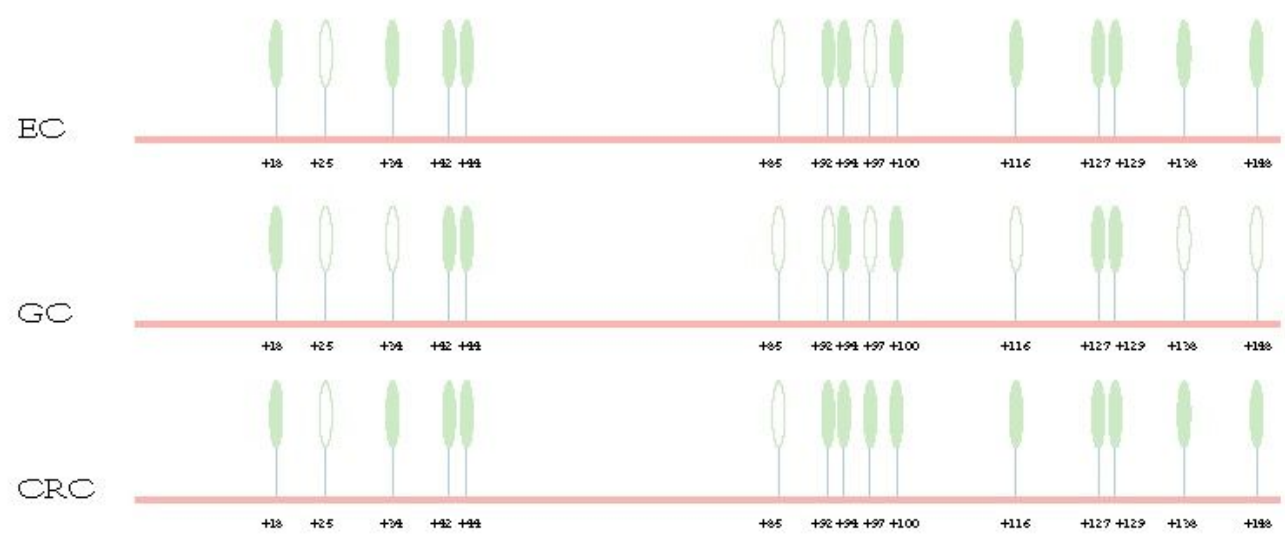

Hyp ermethylation $\quad$ Hypomethylation

Figure 1

CpG sites of SST in gastrointestinal tract cancers.
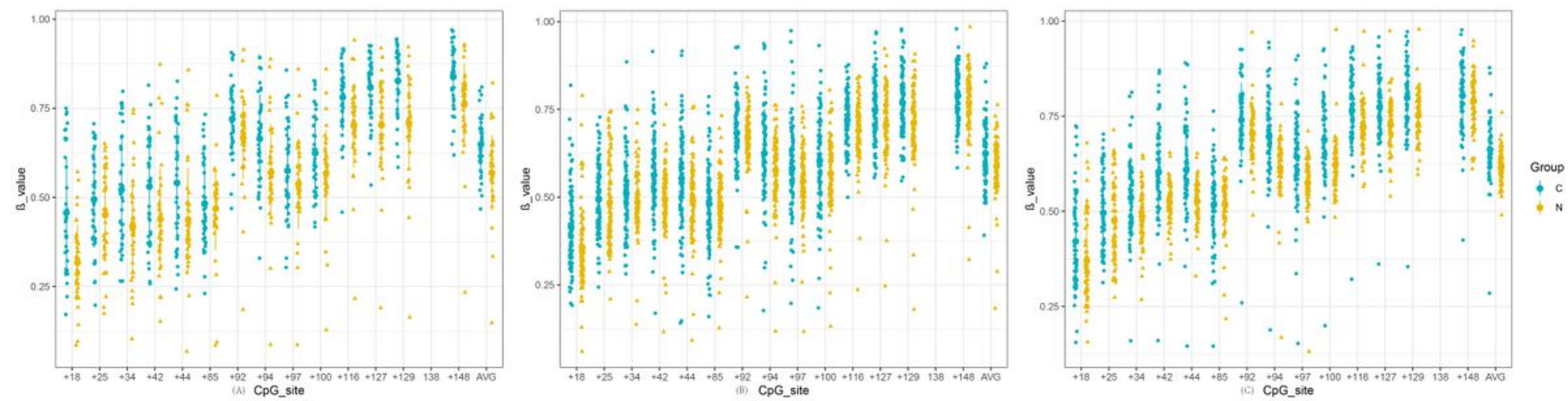

Figure 2

Methylation status of $\mathrm{CpG}$ sites in gastrointestinal tract cancers. (A) EC vs. Control (B) GC vs. Control (C) CC vs. Control. 


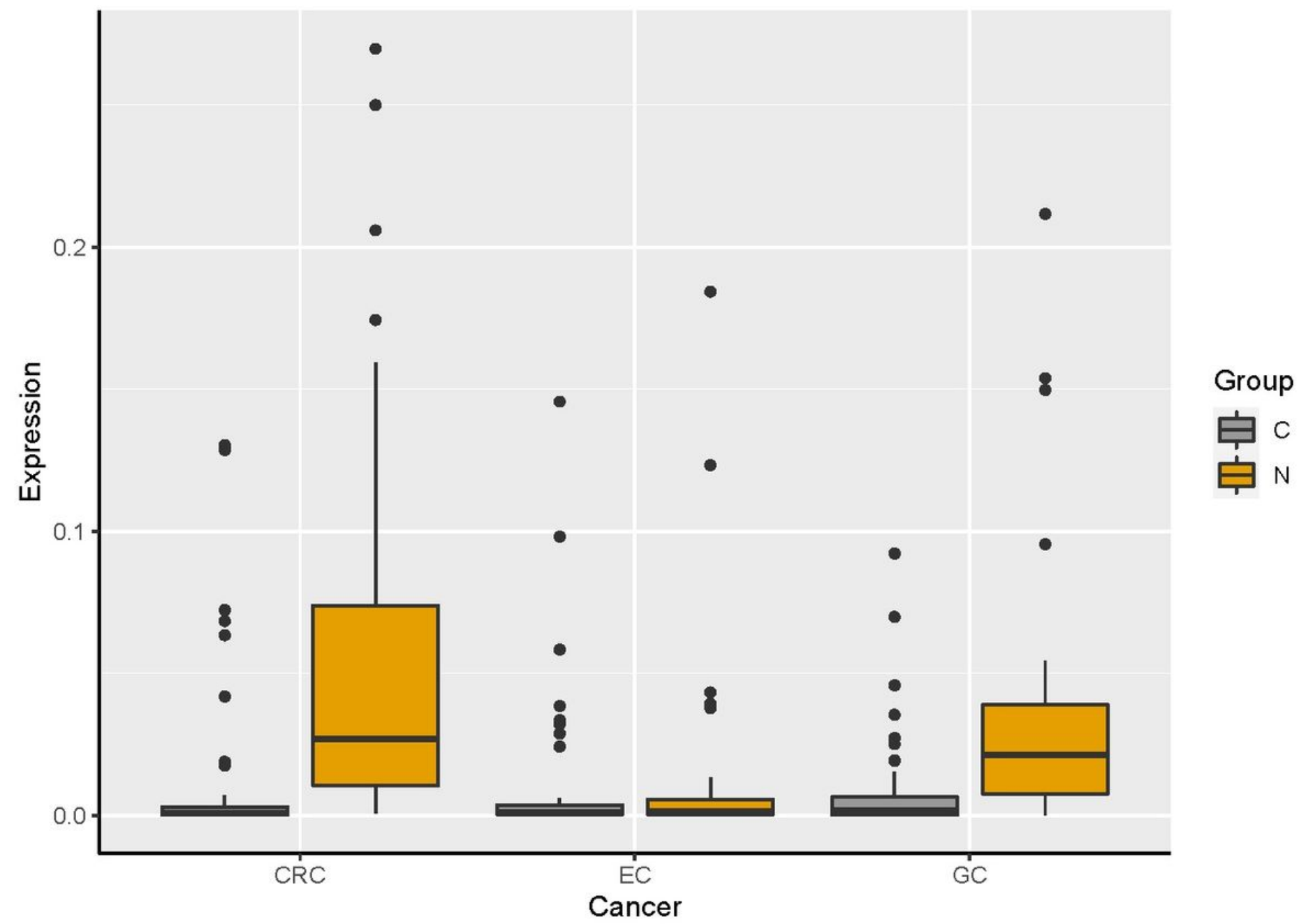

Figure 3

Difference analysis of SST mRNA expression in GIT cancers. 


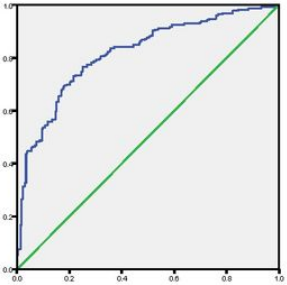

A1
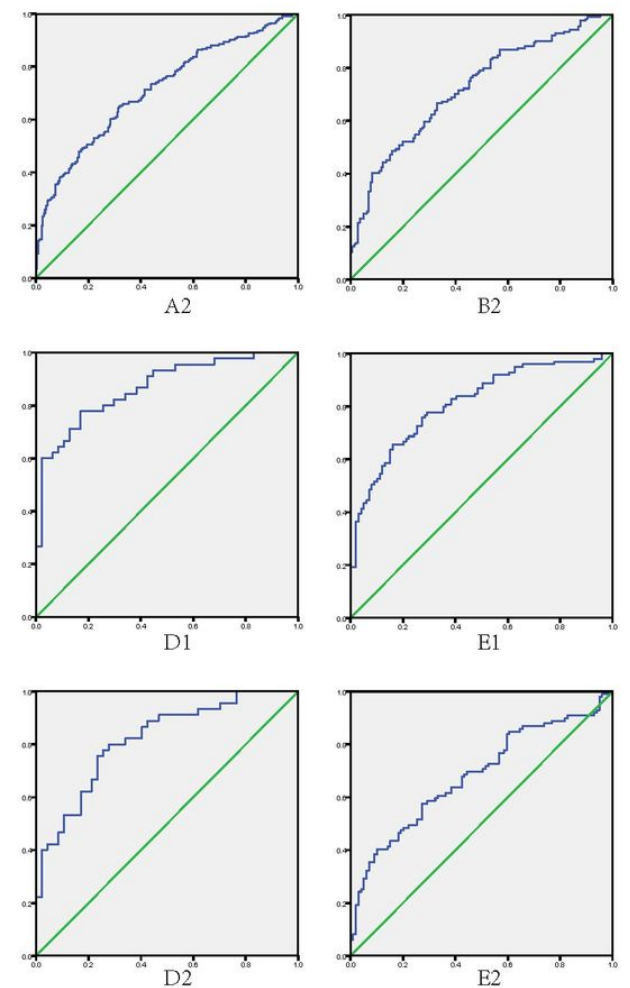
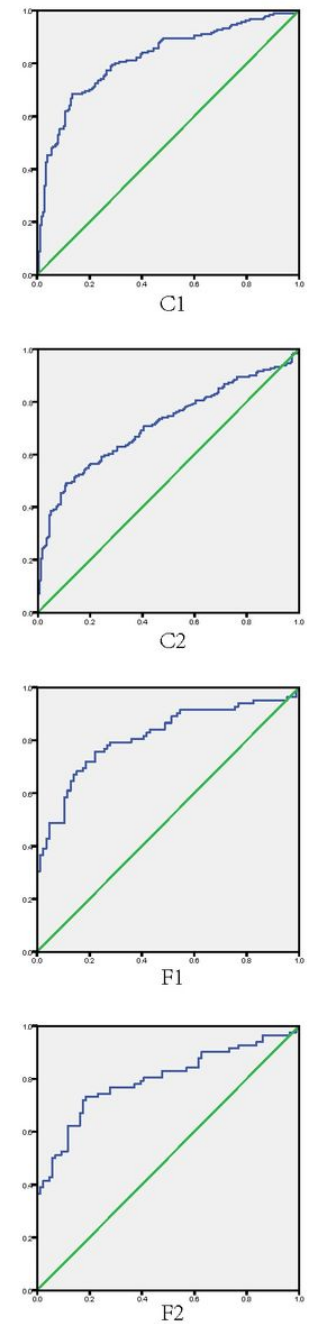

Figure 4

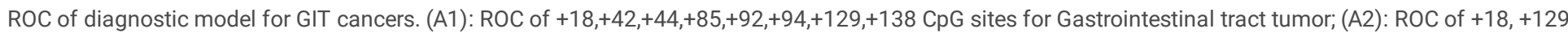

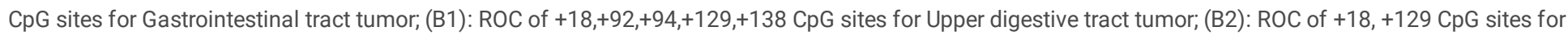
Upper digestive tract tumor; (C1): ROC of $+42,+44,+85,+92,+94,+116,+129,+138$ CpG sites for Gastrointestinal tumor; (C2): ROC of +42 CpG site for

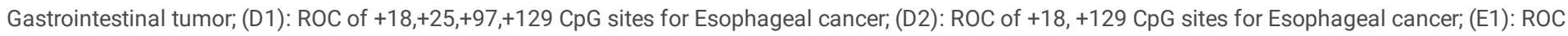

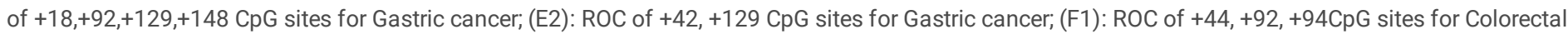
cancer; (F2): ROC of $+44,+94 \mathrm{CpG}$ sites for Colorectal cancer. 